\title{
Comportamientos sexuales y características personales según orientación sexual en adolescentes chilenos
}

\author{
Electra González A. PhD ${ }^{1 a}$, Temístocles Molina G. MSc ${ }^{1 b}$, Jimena San Martin V. ${ }^{2 c}$ \\ ${ }^{1}$ Centro de Medicina Reproductiva y Desarrollo Integral del Adolescente (CEMERA), Facultad de Medicina, Universidad \\ de Chile. ${ }^{2}$ Escuela de Psicología, Universidad Academia de Humanismo Cristiano.
}

${ }^{a}$ Asistente Social. ${ }^{b}$ Estadístico. ${ }^{c}$ Psicóloga.

\section{RESUMEN}

Antecedentes: Los adolescentes y jóvenes lesbianas, gays y bisexuales (LGB) presentan desafíos adicionales en su desarrollo comparados con sus pares heterosexuales, lo cual los expone a altos niveles de estrés y angustia que pueden llevarlos a comportamientos sexuales de riesgo. Objetivo: Analizar los comportamientos sexuales y características personales según la orientación sexual en adolescentes consultantes en un centro de salud sexual y reproductiva. Método: Estudio transversal y analítico en una población adolescente. Los datos fueron recolectados entre 2000-2012. Se utilizó regresión logística múltiple para comparar variables personales, comportamientos sexuales y la orientación sexual. Resultados: En total, 5.143 adolescentes (mediana de edad 16 años, 91,5\% mujeres y $97,5 \%$ estudiantes) fueron estudiados; 127 reportaron orientación LGB. Los adolescentes LGB presentaron riesgo de tener más parejas sexuales en el grupo de adolescentes católicos, y en el grupo total inicio de la actividad sexual más temprana que sus pares heterosexuales, además los adolescentes LGB en el uso de métodos anticonceptivos no mostró ser un factor de riesgo en los adolescentes católicos pero si en los adolescentes con otra o sin religión. Conclusiones: Los resultados de este estudio muestran que los adolescentes LGB y heterosexual no son idénticos en términos de riesgo sexual. La población LGB se compromete en comportamientos sexuales de más riesgo que su contraparte heterosexual. Los proveedores de salud como el sector educación necesitan estar más sensibilizados a estas diferencias y sus implicancias para la salud y consejería de los y las adolescentes.

\section{PALABRAS CLAVE: Adolescente, orientación sexual, comportamiento sexual}

\section{SUMMARY}

Background: Adolescents and young lesbian, gay and bisexual (LGB) present additional challenges in their development compared to their heterosexual peers which exposes them to high levels of stress and distress that can lead to sexual risk behaviors. Aim: To analyze the sexual behavior, personal characteristics and sexual orientation in adolescents that attended in a center of sexual and reproductive health. Methods: Analytical cross-sectional study conducted in an adolescent population. Data were collected in 2000-2012. Multiple logistic regression was used to quantify comparisons of sexual behaviors and personal variables between LGB and heterosexual adolescents. Results: In total, 5,143 adolescents (median age 16 years, $91.5 \%$ women and $97.5 \%$ students) were studied, 127 were LGB teenagers. LGB adolescents reported risk having more sexual partners and sexual debut earlier than their heterosexual peers. This risk increased by catholic teenagers. Using contraception methods showed no risk in catholic teenagers but adolescents assigned to another religion or no religion which was a risk factor. Conclusions: The results of this study show 
that the LGB and heterosexual adolescents are not identical in terms of sexual risk. LGB engages in riskier sexual behaviors than heterosexual counterparts. Healthcare providers and the education sector need to be sensitive to these differences and their implications for health and counseling of adolescents.

\section{KEY WORD: Adolescents, sexual orientation, sexual behavior}

\section{INTRODUCCIÓN}

Las personas lesbianas, gays y bisexuales (LGB) existen y han existido en la mayoría de las sociedades, su prevalencia no se sabe con certeza debido al grado de aceptación que cada sociedad tiene acerca de la orientación no heterosexual, lo cual tiene un efecto directo en los individuos para que oculten o den a conocer su orientación sexual como también, depende si se pregunta por orientación, conducta o identidad sexual. En Estados Unidos la prevalencia varía entre un 3-10\% de población adulta que se declara homosexual (1-3).

En Chile, los datos son aun más escasos. Según la encuesta nacional de comportamiento sexual (CONASIDA; 1998) un $0,3 \%$ de los hombres y un $0,1 \%$ de las mujeres se considera homosexual. Un $0,1 \%$ de los hombres y un $0,1 \%$ de las mujeres se considera bisexual. Según la Encuesta FUNACS (1996) un $2,7 \%$ de los hombres y un 1,1\% de las mujeres declararon actividad sexual con una persona del mismo sexo. El Estudio Nacional de Comportamiento Sexual (4), indica que el $0,3 \%$ de la población reporta mantener relaciones sexuales con personas del mismo sexo. El Censo del 2012 (5) registró que el $0,36 \%$ de los hogares esta constituido por parejas del mismo sexo. Según datos de la última encuesta del INJUV (6), un 2,2\% de la población joven se declara homosexual y un 1,1\% bisexual. Estimaciones sobre la población adolescente se desconoce. Sin embargo a pesar que se desconoce la incidencia real de la orientación homosexual en adolescentes, lo que si se ha observado es que el número de adolescentes que se identifican como homosexuales está aumentando (7).

Si bien los mecanismos de desarrollo de la orientación sexual no están aún completamente aclarados (8) se sabe sobre la influencia biológica en su conformación $(9,10)$. La evidencia científica muestra que ni formas de crianza, tipo de colegio, abuso sexual u otros eventos vitales, causen la orientación sexual homosexual en las personas (11-13). Lo que si ocurre es el hablar del tema de la homosexualidad, el estar expuestos a ver conductas homosexuales, ya sea a través de los distintos medios de comunicación o en distintos ambientes como discotecas, familia, colegio, influye en que las personas homosexuales puedan identificarse con su orientación sexual más precozmente, y en la medida en que el estigma disminuye y el medio social sea más abierto y seguro, puedan con más frecuencia asumirlo públicamente $(14,15)$.

Alrededor de los 12 o 13 años los individuos empiezan a darse cuenta de la atracción hacia miembros de su propio sexo $(16,17)$, y empiezan a incluir la posibilidad de las relaciones homosexuales. Pero al mismo tiempo se dan cuenta que abrir su orientación sexual puede tener un alto costo dado nuestra sociedad heteronormativa, por eso el miedo a ser descubiertos los lleva a disminuir su participación en actividades sociales con pares de su mismo sexo (18). Sienten que no pueden compartir sentimientos con pares de su mismo sexo y entonces empiezan a alienarse tanto física como emocionalmente (19). Algunos responden intentando algún acercamiento amoroso con el sexo opuesto para probar sentimientos sexuales y acomodarse con sus pares. Pero esta situación puede generarles sentimientos de soledad, frustración, sentir que son un fraude y sentimientos de no pertenencia (13).

Si bien los adolescentes y jóvenes LGB presentan los mismos cambios del desarrollo que sus pares heterosexuales deben enfrentar desafíos adicionales que tiene que ver con su orientación distinta a la heteronormatividad y con el proceso de reconocerse y aceptarse como una persona LGB. Todo esto los expone a altos niveles de angustia y estrés psicológico que pueden llevarlos a comportamientos de riesgo. Más aun se ven enfrentados a tener que acudir a servicios sociales, de salud y educacionales que responden a esta cultura heteronormativa.

La evidencia muestra que la población adolescente LGB presenta indicadores de riesgo más altos que sus pares heterosexuales. En salud mental presentan de 3 a 5 veces más probabilidad de consumo de drogas y alcohol (20), 3 veces más probabilidad de ideación suicida o intento suicida (20). En salud sexual, mayor prevalencia de VIH/SIDA, otras infecciones de transmisión sexual, mayor probabilidad de tener más parejas sexuales e inicio de actividad sexual a edades más tempranas que adolescentes heterosexuales (21), como también menos uso de condón durante el último coito con parejas heterosexuales (35,8\% vs $65,5 \%$ ) (22) y se comprometen en prácticas sexuales más riesgosas 
(23). En salud reproductiva se ha reportado mayor frecuencia de embarazo en adolescentes lesbianas y bisexuales (12\% vs 5\%) (21-23). En relación a violencia sexual, jóvenes LGB reportan un $33 \%$ vs $9 \%$ de contacto sexual en contra de su voluntad (22). Un $85 \%$ reportó haber sido víctima de acoso verbal, un $40 \%$ experimentó acoso físico y dos tercios reportaron sentirse inseguros en sus colegios. En nuestro país prácticamente no existen publicaciones científicas que aborden esta problemática en adolescentes.

El objetivo de este estudio fue analizar los comportamientos sexuales y características personales según la orientación sexual en adolescentes consultantes en un centro universitario de salud sexual y reproductiva.

\section{SUJETOS Y MÉTODO}

Diseño: Estudio transversal y analítico en adolescentes que fueron atendidos en el Centro de Medicina Reproductiva y Desarrollo Integral del Adolescente (CEMERA) durante el período 2000-2012. Durante estos años 5.143 adolescentes consultaron en CEMERA. Este estudio consistió en una primera etapa en describir la asociación entre dos grupos: uno conformado por adolescentes heterosexuales y otro por adolescentes LGB y variables personales y familiares. En una segunda etapa se ajustaron tres modelos, considerando como variables dependientes los comportamientos sexuales número de parejas sexuales, uso de métodos anticonceptivos (MAC) y edad de inicio de actividad sexual y como variables independientes orientación sexual y variables personales seleccionadas.

Comportamientos sexuales: Número de parejas sexuales dicotomizada como: una pareja $=0$, dos y más=1. Uso de anticoncepción al inicio de actividad sexual codificada como: no=1, sí=0. Edad inicio actividad sexual: dicotomizada como: 10 a 15 años $=1$, de 16 a 19 años=0

Variables personales: Orientación sexual dicotomizada como: heterosexual=0, homosexual, lesbiana, bisexual=1. Género: codificada como: mujer $=0$, hombre $=1$. Edad. Escolaridad: se hicieron variables dummy, categoría de referencia= Superior. Promedio de notas. Religión: se hicieron variables dummy, categoría de referencia= Católica. Planes futuros: se hicieron variables dummy, categoría de referencia= Enseñanza superior. Con quién conversa temas de sexualidad: se hicieron variables dummy, categoría de referencia $=$ Padres y familiares. Participación social: codificada como: participa $=0$, no participa $=1$.

Variables familiares: Supervisión en la semana (con horario, sin horario, no sale), Supervisión fin de semana (con horario, sin horario, no sale),
Escolaridad madre (básica, media superior), APGAR familiar (funcional, disfuncional), Relación familiar (buena, regular, mala), Comunicación familiar (buena, regular, mala), Nivel socio-económico (24) (alto, medio, bajo), Filiación (matrimonial, no matrimonial), Número de hermanos, Número de orden dentro de los hermanos, Quien supervisa (ambos padres, padre, madre, otros), Castigo (grave, moderado, sin sanción) y Escolaridad padre (básica, media superior).

Para medir la asociación entre las variables personales, familiares y la orientación sexual se utilizó las pruebas estadísticas Chi-cuadrado, $t$ de Student y Wilcoxon para 2 muestras independientes.

Para medir la asociación entre las variables de comportamiento sexual (edad inicio actividad sexual, uso de anticoncepción al inicio de actividad sexual, número de parejas sexuales), y las variables de orientación sexual y personales, primero se evaluó la existencia de variables de confusión y modificadoras de efecto (interacción) y posteriormente se utilizó regresión logística múltiple (25), para cada comportamiento de riesgo. En el caso que exista variables modificadoras de efecto, los modelos se analizaran para cada categoría de esta variable. La bondad de ajuste, se midió con la prueba de Hosmer-Lemeshow, curva ROC y los criterios de clasificación Akaike (AIC) y Bayesiano (BIC) (25). Se utilizó el software estadístico STATA 12.0 (StataCorp LP, Texas, USA)

La confidencialidad y anonimato de los datos fue formalizado mediante firma del consentimiento informado por los adolecentes. El estudio fue aprobado por el Comité de Ética de Investigación en Seres Humanos de la Facultad de Medicina.

\section{RESULTADOS}

Consultaron en el período de estudio 5.143 adolescentes. De los cuales 127 corresponden a adolescentes con orientación LGB y 5.016 adolescentes heterosexuales; $4.708(91,5 \%)$ mujeres y $435(8,5 \%)$ varones.

La mediana de edad fue 16 años (rango: 10 a 19), estudiantes el $97,3 \%$, el rendimiento escolar alcanzó un promedio de 5,5. El 45,5\% participaba en actividades sociales comunitarias, la afiliación religiosa reportada fue de $81 \%$. El $65,6 \%$ tenía como aspiraciones académicas alcanzar estudios superiores. La mediana de edad de inicio de actividad sexual fue de 15 años (rango: 10 a 19 años)

El análisis bivariado mostró que los comportamientos sexuales de riesgo asociados a la orientación sexual LGB fueron: la edad de inicio de la actividad sexual antes de los 16 años y tener 2 o más parejas mientras que el uso de MAC no fue asociado a la orientación sexual (Tabla I). 
Tabla I

DISTRIBUCION DE COMPORTAMIENTOS SEXUALES SEGÚN ORIENTACION SEXUAL

\begin{tabular}{|c|c|c|c|c|}
\hline \multirow[b]{2}{*}{ Variable comportamientos sexuales } & & \multicolumn{3}{|c|}{ Orientación sexual } \\
\hline & & $\begin{array}{c}\text { Heterosexual } \\
\mathrm{n}(\%)\end{array}$ & & $\begin{array}{l}\text { LGB } \\
\mathrm{n}(\%)\end{array}$ \\
\hline \multirow[t]{4}{*}{ Edad inicio actividad sexual } & 10 a 15 & $2164(53,8)$ & & $38(74,5)$ \\
\hline & 16 a 19 & $1860(46,2)$ & & $13(25,5)^{\star}$ \\
\hline & Total & $4024(100)$ & & $51(100)$ \\
\hline & $\mathrm{p}$ & & 0,003 & \\
\hline \multirow[t]{4}{*}{ Uso de MAC } & $\mathrm{Si}$ & $2504(62,6)$ & & $23(51,1)$ \\
\hline & No & $1495(37,4)$ & & $22(48,9)$ \\
\hline & Total & $3999(100)$ & & $45(100)$ \\
\hline & $\mathrm{p}$ & & 0,11 & \\
\hline \multirow[t]{4}{*}{ Número de parejas sexuales } & Una & $2900(72,1)$ & & $21(42,0)$ \\
\hline & Dos y más & $1124(27,9)$ & & $29(58,0)^{\star}$ \\
\hline & Total & $4024(100)$ & & $50(100)$ \\
\hline & $\mathrm{p}$ & & 0,001 & \\
\hline
\end{tabular}

Al analizar las variables personales asociadas a la orientación sexual se observó que los adolescentes LGB con mayor frecuencia son de sexo masculino (47,2\% versus $7,5 \%)$; se definen sin religión ( $40 \%$ versus $18,5 \%$ ); tienen menos edad (15,7 versus 16 años) y conversan temas de sexualidad con "otros o nadie" (19,2\% versus $7,6 \%)$, mientras que tener como planes futuros solo terminar enseñanza media se observó más frecuentemente en los adolescentes heterosexuales, (26,4\% versus $18,3 \%)$; escolaridad, participación social y promedio de notas no mostró diferencias al analizar por orientación sexual (Tabla II).

Tabla II

DISTRIBUCION DE LAS VARIABLES PERSONALES SEGÚN ORIENTACION SEXUAL

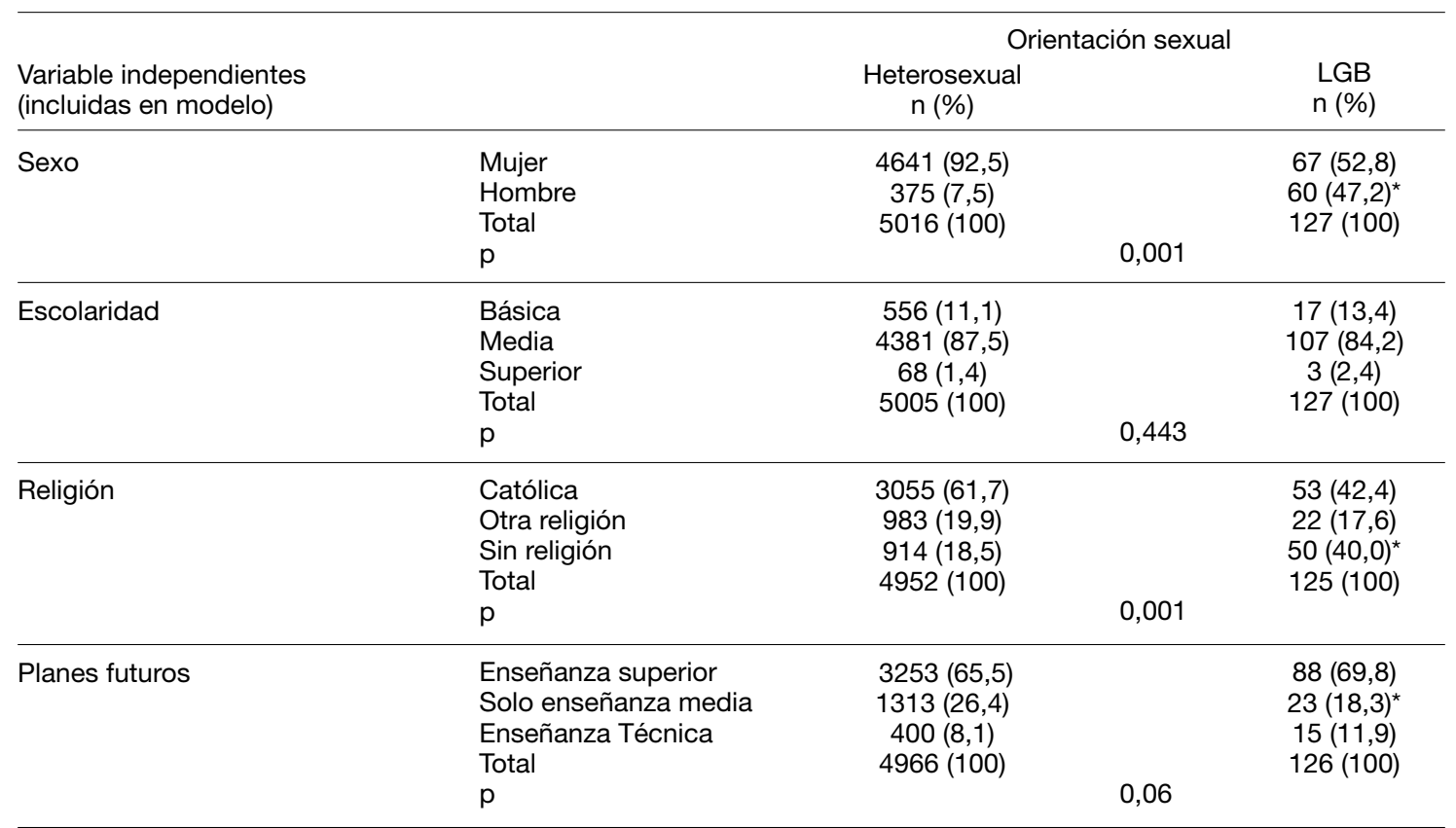




\section{CONTINUACIÓN TABLA II}

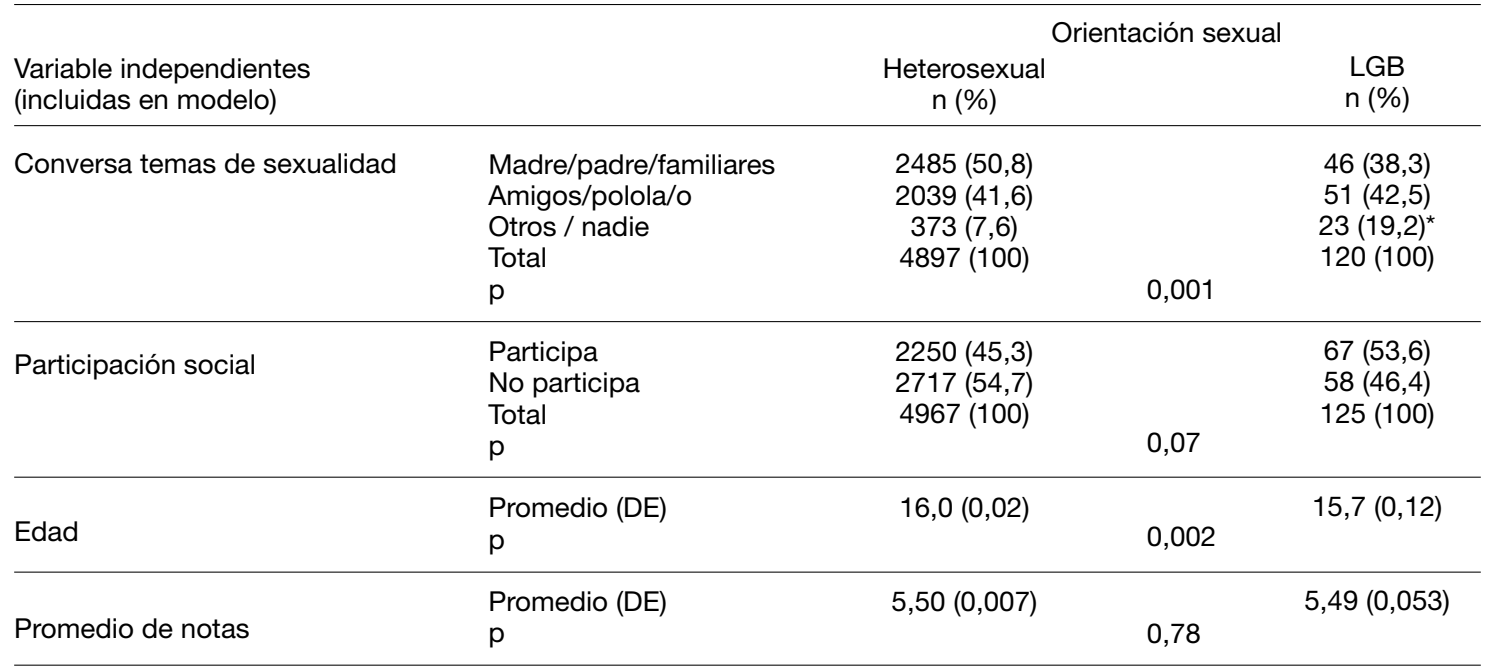

Al analizar las variables familiares asociadas a la orientación sexual se observó que los adolescentes con orientación LGB reportaron con mayor frecuencia que no salen el fin de semana $(36,8 \%$ y $13,4 \%)$, escolaridad materna superior (15\% y $8,1 \%)$, mala comunicación familiar $(25,4 \%$ y $17,7 \%)$ y NSE alto $(16,7 \%$ y $4,5 \%)$ mientras que los adolescentes heterosexuales reportaron mayor frecuencia en supervisión con horario durante la semana y buena relación familiar (49\% y 34,7\%) (Tabla III). Debido que la variable Religión resulto ser una variable modificadora de efecto, los modelos de regresión logística múltiple se analizaron para cada categoría de esta variable.

\section{Tabla III}

\section{DISTRIBUCION DE LAS VARIABLES FAMILIARES SEGÚN ORIENTACION SEXUAL}

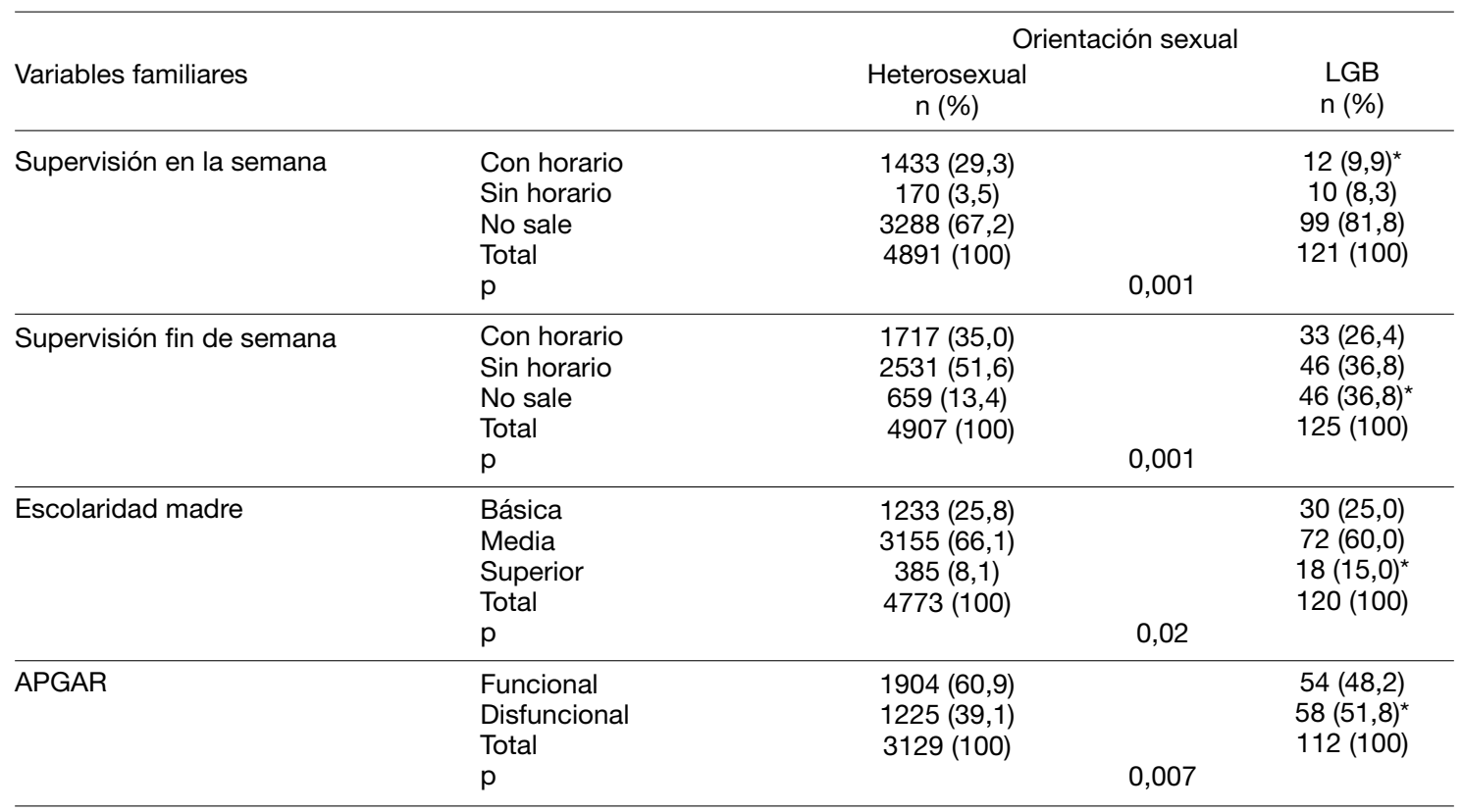




\section{CONTINUACIÓN TABLA III}

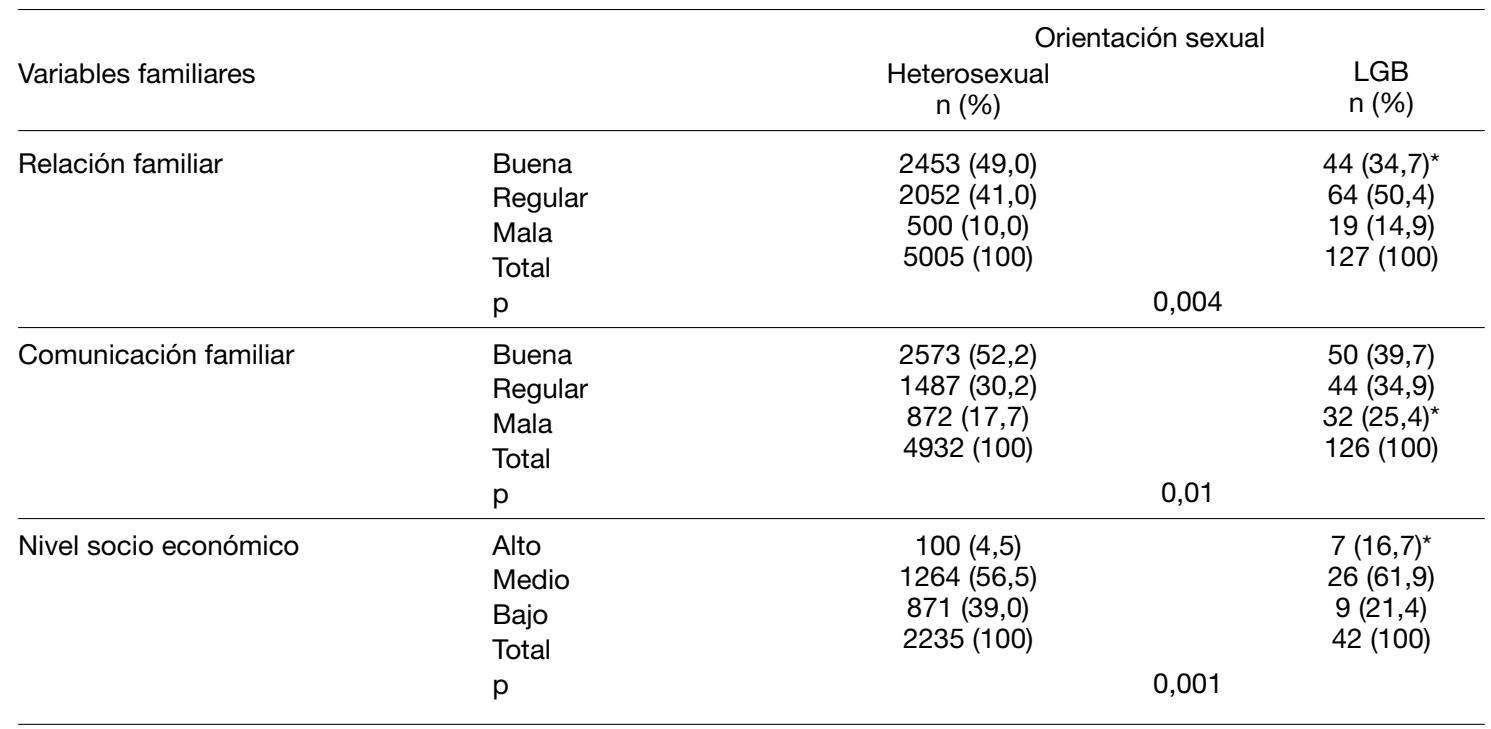

En el $1^{\circ}$ Modelo Número de parejas sexuales en adolescentes católicos resultaron significativas las variables: Orientación sexual LGB, incrementó el riesgo en 4,22 veces más de tener más de una pareja sexual (OR 5,22); Sexo masculino, incrementó el riesgo en un $92 \%$ más de tener más de una pareja sexual (OR 1,92); Edad, por cada año más de edad que tenga el adolescente, se incrementó el riesgo en un $23 \%$ (OR 1,23$)$. Mientras que Promedio de notas, por cada punto más en el promedio de notas, disminuyó en un $33 \%$ tener más de una pareja sexual $(O R 0,67)(p<0,05)$ controlado por el resto de las variables (Tabla IV).

En adolescentes con otra religión: Sexo masculino, incrementó el riesgo 1,22 veces de tener más de una pareja sexual (OR 2,22). Edad, por cada año más de edad, se incrementó el riesgo en un $20 \%$ (OR 1,20). Promedio de notas, por cada punto más en el promedio de notas, disminuyó el riesgo en un $31 \%(\mathrm{OR} 0,69)(p<0,05)$ controlado por el resto de las variables. (Tabla IV)

En adolescentes sin religión fueron: Sexo masculino, se incrementa el riesgo un 57\% (OR 1,57). Edad, por cada año más de edad, se incrementó el riesgo en un $30 \%$ (OR 1,30). Promedio de notas, por cada punto más en el promedio de notas que tenga el adolescente, disminuyó el riesgo en un $41 \%(0,59)(p<0,05)$ controlado por el resto de las variables (Tabla IV).
En el $2^{\circ}$ Modelo Uso de MAC en adolescentes católicos resultaron significativas las variables: Edad, por cada año más de edad, disminuyó el riesgo en un $14 \%$ de no usar MAC (OR 0,86); Promedio de notas, por cada punto más en el promedio de notas, disminuye el riesgo en un 33\% (OR $0,67)$; Planes de estudio futuros solo terminar enseñanza media incrementó el riesgo en un 30\% (OR 1,30); Conversa temas de sexualidad, con amigos o pololos incrementó el riesgo en un 37\% (OR 1,37); Conversar temas de sexualidad con otros 0 nadie incrementó el riesgo en un $79 \%$ (OR 1,79) $(p<0,05)$ controlado por el resto de las variables (Tabla V).

En adolescentes con otra religión la Orientación sexual LGB, incrementó el riesgo en 14,3 veces no usar MAC (OR 14,3). Escolaridad básica, incrementa el riesgo en 10,1 veces (OR 10,1). Escolaridad media, incrementó el riesgo en 2,81 veces (OR 2,81). Promedio de notas, por cada punto más en el promedio de notas que tenga el adolescente, disminuye el riesgo en un $31 \%(\mathrm{OR} 0,69)(p<0,05)$ controlado por el resto de las variables (Tabla V).

En adolescentes sin religión la Orientación sexual LGB, incrementó el riesgo en 2,54 veces no usar MAC (OR 2,54); Edad, por cada año más, disminuye en un $18 \%$ (OR 0,82); Promedio de notas, por cada punto más en el promedio de notas, disminuye el riesgo en un $37 \%(O R 0,63)(p<0,05)$ controlado por el resto de las variables (Tabla V). 


\section{REGRESION LOGISTICA MULTIPLE ENTRE NUMERO DE PAREJAS SEXUALES Y ORIENTACION SEXUAL, VARIABLES PERSONALES SEGÚN RELIGION}

\begin{tabular}{|c|c|c|c|c|}
\hline Religión & Variable & Odds Ratio & Valor $\mathrm{p}$ & IC95\% \\
\hline \multirow[t]{7}{*}{ Católica } & Orientación sexual & 5,22 & 0,003 & $1,77-15,36$ \\
\hline & Sexo & 1,92 & 0,0001 & $1,37-2,68$ \\
\hline & Edad & 1,23 & 0,0001 & $1,13-1,33$ \\
\hline & Promedio de notas & 0,67 & 0,0001 & $0,55-0,81$ \\
\hline & Hosmer-Lemeshow: 0,75; Curva ROC: 0,60 & & & \\
\hline & AIC modelo sencillo: 2793,8; AIC modelo completo: 2668 & & & \\
\hline & BIC modelo sencillo: 2805,4; BIC modelo completo: 2696,9 & & & \\
\hline \multirow[t]{7}{*}{ Otra religión } & Orientación sexual & 3,34 & 0,103 & $0,78-14,26$ \\
\hline & Sexo & 2,22 & 0,003 & $1,31-3,74$ \\
\hline & Edad & 1,20 & 0,014 & $1,04-1,39$ \\
\hline & Promedio de notas & 0,69 & 0,024 & $0,49-0,95$ \\
\hline & Hosmer-Lemeshow: 0,97; Curva ROC: 0,62 & & & \\
\hline & AIC modelo sencillo: 912,9 ; AIC modelo completo: 855,9 & & & \\
\hline & BIC modelo sencillo: 922,3; BIC modelo completo: 879 & & & \\
\hline \multirow[t]{7}{*}{ Sin religión } & Orientación sexual & 1,42 & 0,43 & $0,59-3,41$ \\
\hline & Sexo & 1,57 & 0,053 & $0,99-2,47$ \\
\hline & Edad & 1,30 & 0,0001 & $1,14-1,49$ \\
\hline & Promedio de notas & 0,59 & 0,0001 & $0,44-0,78$ \\
\hline & Hosmer-Lemeshow: 0,6; Curva ROC: 0,61 & & & \\
\hline & AIC modelo sencillo: 1041; AIC modelo completo: 981,9 & & & \\
\hline & BIC modelo sencillo: 1050,3; BIC modelo completo: 1005,2 & & & \\
\hline
\end{tabular}

Tabla V

REGRESION LOGISTICA MULTIPLE ENTRE USO DE MAC Y ORIENTACION SEXUAL, VARIABLES PERSONALES SEGÚN RELIGION

\begin{tabular}{|c|c|c|c|c|}
\hline Religión & Variable & Odds Ratio & Valor $p$ & IC95\% \\
\hline \multirow[t]{11}{*}{ Católica } & Orientación sexual & 0,45 & 0,229 & $1,12-1,65$ \\
\hline & Edad & 0,86 & 0,001 & $0,80-0,92$ \\
\hline & Promedio de notas & 0,67 & 0,001 & $0,56-0,79$ \\
\hline & Planes futuros (solo EM) & 1,30 & 0,008 & $1,07-1,59$ \\
\hline & Planes futuros (Técnico) ${ }^{1}$ & 1,13 & 0,46 & $0,81-1,57$ \\
\hline & Conversa temas sexuales Amigos/pololo 2 & 1,37 & 0,001 & $1,16-1,65$ \\
\hline & Conversa temas sex. Otros nadie ${ }^{2}$ & 1,79 & 0,001 & $1,27-2,53$ \\
\hline & 1: Planes futuros: categoría de referencia: enseñanza universitaria & & & \\
\hline & $\begin{array}{l}\text { 2: Conversa temas de sex.: categoría de referencia: madre/padre/familiares } \\
\text { Hosmer-Lemeshow: } 0,04 \text {; Curva ROC: } 0,60\end{array}$ & & & \\
\hline & AIC modelo sencillo: 3237,$4 ;$ AIC modelo completo: 3046,1 & & & \\
\hline & BIC modelo sencillo: 3249; BIC modelo completo: 3092,2 & & & \\
\hline \multirow[t]{7}{*}{ Otra religión } & Orientación sexual & 14,3 & 0,012 & $1,78-115,64$ \\
\hline & Escolaridad (básica) & 10,1 & 0,005 & $1,99-51,44$ \\
\hline & Escolaridad (media) & 2,81 & 0,18 & $0,62-12,83$ \\
\hline & Promedio de notas & 0,69 & 0,02 & $0,52-0,94$ \\
\hline & Hosmer-Lemeshow: 0,86; Curva ROC: 0,61 & & & \\
\hline & AIC modelo sencillo: $1031 ;$ AIC modelo completo: 970,4 & & & \\
\hline & BIC modelo sencillo: 1040,3; BIC modelo completo: 993,5 & & & \\
\hline
\end{tabular}




\section{CONTINUACIÓN TABLA V}

\begin{tabular}{llrrr}
\hline Religión & Variable & Odds Ratio & Valor $\mathrm{p}$ & IC95\% \\
\hline \multirow{2}{*}{ Sin religión } & Orientación sexual & 2,54 & 0,049 & $1,003-6,44$ \\
& Edad & 0,82 & 0,001 & $0,72-0,92$ \\
& Promedio de notas & 0,63 & 0,001 & $0,47-0,83$
\end{tabular}

Hosmer-Lemeshow: 0,08; Curva ROC: 0,61

AIC modelo sencillo: 1018,7; AIC modelo completo: 961

BIC modelo sencillo: 1028; BIC modelo completo: 979,5

En el $3^{\circ}$ Modelo Edad inicio actividad sexual las variables que resultaron significativas fueron: Orientación sexual LGB,incrementó el riesgo en 1,55 veces más de iniciar actividad sexual antes de los 16 años; Sexo masculino incrementó el riesgo 2,11 veces; Edad, por cada año más de edad, disminuye el riesgo en un $77 \%$; Promedio de notas, por cada punto más en el promedio de notas, disminuye el riesgo en un $23 \%$; Planes de estudio futuros solo enseñanza media incrementa el riesgo en un $21 \%$ (OR 1,21) $(p<0,05)$ controlado por el resto de las variables (Tabla $\mathrm{VI}$ ).

\section{DISCUSIÓN}

Este estudio mostró diferencias en los comportamientos sexuales de adolescentes heterosexuales y adolescentes LGB, lo que es concordante con lo reportado por estudios internacionales (21-23).

El riesgo de tener más parejas sexuales se incremento en adolescentes LGB que reportaron ser católicos. En los adolescentes que se adscribieron a otra religión o se definieron como sin religión la orientación sexual no mostró diferencias. Los adolescentes varones presentaron mayor riesgo. En los tres grupos los adolescentes de mayor edad presentaron más riesgo. El mejor promedio de notas resulto ser un factor protector.

Los adolescentes LGB, varones y con planes futuros solo enseñanza media presentaron más riesgo de inicio sexual temprano. La edad y promedio de notas resultaron ser un factor protector.

Llama la atención que el riesgo de no usar MAC aumenta en adolescentes LGB que reportaron otra religión o sin religión y no en los que se definieron como católicos. El promedio de notas fue un factor protector en los tres grupos y la edad en los católicos y sin religión. En los católicos conversar temas de sexualidad con amigos/pololo u otros/ aumenta el riesgo de no uso de MAC.

El estudio tiene importantes implicancias tanto para el sector salud como de educación. Los programas de educación sexual escolar debieran enseñar prácticas sexuales seguras para todos los estudiantes que tienen actividad sexual considerando todas las orientaciones sexuales. Eso significa enseñar prevención de embarazo y habilidades en la negociación del condón para adolescentes

\section{Tabla VI \\ REGRESION LOGISTICA MULTIPLE ENTRE EDAD DE INICIO DE ACTIVIDAD SEXUAL Y VARIABLES PERSONALES}

\begin{tabular}{lccc}
\hline Variable & Odds Ratio & Valor p & IC95\% \\
\hline Orientación sexual & 2,55 & 0,015 & $1,20-5,42$ \\
Sexo & 3,11 & 0,0001 & $2,31-4,20$ \\
Edad & 0,23 & 0,0001 & $0,21-0,26$ \\
Promedio de notas $_{\text {Planes futuros: solo enseñanza media }}{ }^{1}$ & 0,77 & 0,001 & $0,66-0,90$ \\
Planes futuros: enseñanza técnica $^{1}$ & 1,21 & 0,046 & $1,003-1,44$ \\
& 0,90 & 0,489 & $0,67-1,21$
\end{tabular}

1: Planes futuros: categoría de referencia: enseñanza universitaria

Hosmer-Lemeshow: 0,001; Curva ROC: 0,83

AIC modelo sencillo: 5617,4; AIC modelo completo: 3896,4

BIC modelo sencillo: 5630; BIC modelo completo: 3940,4 
LGB como heterosexuales, tanto católicos como no católicos.

Limitaciones del estudio: Es un estudio transversal lo que impide conocer la relación de causalidad.

\section{CONCLUSIÓN}

Los y las adolescentes no son idénticos en términos de riesgo sexual. Esto debe ser tomado en cuenta por los proveedores de salud como por el sector educación para ser más sensibles a estas diferencias y sus implicancias para la salud y consejería en adolescentes.

\section{REFERENCIAS}

1. American Academy of Pediatrics, Committee on Psychosocial Aspects of Child and Family Health and Committee on Adolescence. Sexuality education for children and adolescents. Pediatrics 2001;108:498502.

2. American Academy of Pediatrics. Confidentiality in adolescent health care. AAP News. April 1989:9. Reaffirmed January 1993

3. Black D, Gates G, Sanders S, Taylor L. Demographics of the gay and lesbian population in the United States: Evidence from available systematic data sources. Demography 2000;37(2):139-54.

4. Estudio Nacional de Comportamiento Sexual. Síntesis de Información seleccionada. Chile. MINSAL. 2000.

5. Censo 2012. Instituto Nacional de Estadísticas. Chile.

6. VII Encuesta INJUV. 2012.

7. Frankowski BL, American Academy of Pediatrics Committee on Adolescence. Sexual orientation and adolescents. Pediatrics 2004;113(6):1827-3.

8. Division 44/Committee on Lesbian, Gay, and Bisexual Concerns Joint Task Force on Guidelines for Psychotherapy with Lesbian, Gay, and Bisexual Clients. Guidelines for psychotherapy with lesbian, gay, and bisexual clients. Am Psychol 2000;55(12):144051.

9. Stoller RJ, Herdt GH. Theories of Origins of Male Homosexuality. Arch Gen Psychiatry 1985;42:399-404.

10. Garofalo R, Wolf RC, Wissow LS, Woods ER, Goodman E. Sexual orientation and risk of suicide attempts among a representative sample of youth. Arch Pediatr Adolesc Med 1999;153:487-93.
11. Garofalo R, Wolf RC, Kessel S, Palfrey SJ, DuRant $\mathrm{RH}$. The association between health risk behaviors and sexual orientation among a school-based sample of adolescents. Pediatrics 1998;101:895-902.

12. Remafedi G, French S, Story M, Resnick MD, and Blum R. The relationship between suicide risk and sexual orientation: results of a population-based study. Am J Public Health 1998;88:57-60.

13. Russell ST, Franz BT, Driscoll AK. Same-sex romantic attraction and experiences of violence in adolescence. Am J Public Health 2001;91:903-6

14. Savin-Williams RC, Dubé EM. Parental Reactions to Their Child's Disclosure of a Gay/Lesbian Identity. Family Relations 1998;47:7-13.

15. Anderson D. Family and peers relation of gay adolescents. Adolescent Psichiatry 1987;14:162-78.

16. De Monteflores C, Schultz SJ. Coming out: Similarities and differences for lesbian and gay men. J Social Issues 1978;34:59-72.

17. Hetrick ES, Martin AD. Developmental issues and their resolution for gay and lesbian adolescents. J Homosexuality 1987;14:25-43.

18. Troiden RR. The formation of sexual identities. J Homosexuality 1989;17:43-73.

19. Marshal M, Friedman M, Stall R, King K, Milers J, Sexual orientation and adolescent substance use: meta analysis and methodological review. Addiction 2008;103:546-56.

20. Tumaini R, Austin S, Schuster M. The health and health care of LGB adolescents. Annu Rev Public Health 2010;31:457-77.

21. Levine D. Office-based care for lesbian, gay, bisexual, transgender and questioning youth. Pediatrics 2013;132:297-313.

22. Recommendations for promoting the health and wellbeing of lesbians, gay, bisexual, and transgender adolescents: A position paper of the Society for Adolescent. J Adolesc Health 2013;52:506-10.

23. Ybarra M, Rosario M, Saewyc E, Goodenow C. Sexual behaviors and partners characteristics by sexual identity among adolescent girls. J Adolesc Health 2016;58:310-6.

24. Alvarez M, Muzzo S, Ivanovic D. Escala para medición del nivel socioeconómico en el área de salud. Rev Med Chile 1985;113:243-9.

25. Hosmer DW, Lemeshow S. Applied Logistic Regression, 2nd Edition; New York: John Wiley \& Sons. INC, 2000. 